PROCEEDINGS OF THE

AMERICAN MATHEMATICAL SOCIETY

Volume 132, Number 12, Pages 3457-3462

S 0002-9939(04)07031-5

Article electronically published on July 14, 2004

\title{
PARTITION IDENTITIES AND GEOMETRIC BIJECTIONS
}

\author{
IGOR PAK
}

(Communicated by John R. Stembridge)

\begin{abstract}
We present a geometric framework for a class of partition identities. We show that there exists a unique bijection proving these identities, which satisfies certain linearity conditions. In particular, we show that Corteel's bijection enumerating partitions with nonnegative $r$-th differences can be obtained by our approach. Other examples and generalizations are presented.
\end{abstract}

\section{INTRODUCTION}

Let $\lambda=\left(\lambda_{1}, \ldots, \lambda_{k}\right), \lambda_{1} \geq \cdots \geq \lambda_{k} \geq 1$, be a partition of $n$, denoted by $\lambda \vdash n$. Define the first differences by $\left(\Delta_{1}^{1}(\lambda), \Delta_{2}^{1}(\lambda), \ldots\right)=\left(\lambda_{1}-\lambda_{2}, \lambda_{2}-\lambda_{3}, \ldots\right)$, the second differences by $\left(\Delta_{1}^{2}(\lambda), \Delta_{2}^{2}(\lambda), \ldots\right)=\left(\Delta_{1}^{1}(\lambda)-\Delta_{2}^{1}(\lambda), \Delta_{2}^{1}(\lambda)-\Delta_{2}^{1}(\lambda), \ldots\right)$, etc. Finally, define the $r$-th differences by $\left(\Delta_{1}^{r}(\lambda), \Delta_{2}^{r}(\lambda), \ldots\right)=\left(\Delta_{1}^{r-1}(\lambda)-\Delta_{2}^{r-1}(\lambda)\right.$, $\left.\Delta_{2}^{r-1}(\lambda)-\Delta_{3}^{r-1}(\lambda), \ldots\right)$.

Andrews showed [A3] (see also [APR]) that the number of partitions of $n$ with nonnegative $r$-th differences is equal to the number of partitions of $n$ into parts of the form $\left(\begin{array}{l}i \\ r\end{array}\right)$, where $i \geq r$. This result was discovered with the help of Andrews, Paule and Riese's Omega package [APR]. It was proved combinatorially by Corteel [CCH], Z] by the following simple bijection: $\sigma: \lambda \rightarrow\left\{\left(\begin{array}{c}r \\ r\end{array}\right)^{\Delta_{1}^{r}(\lambda)}\left(\begin{array}{c}r+1 \\ r\end{array}\right)^{\Delta_{2}^{r}(\lambda)} \ldots\right\}$. Not shy on adjectives, Zeilberger [Z] called Andrews's Theorem "elegant", and referred to Corteel's bijection as "brilliant", "human-generated" (sic), "one-line-proof", and "natural" (emphasis follows [Z]). The purpose of this note is to make formal the notion of a "natural bijection". We restate Andrews's Theorem in a geometric setting and present the proof. Corteel's bijection becomes a simple corollary of the setup, unique in a certain precise sense. We then extend the approach to a wide class of related partition results, in the spirit of a recent paper by Corteel and Savage [CS].

Following the structure of [A3], we first tackle a classical problem of enumeration of integer triangles, where we exhibit how a geometric setting completely resolves the problem. We then switch to Andrews's Theorem and to Corteel-Savage type generalizations. We conclude the paper with examples and final remarks.

Let us emphasize that at no point do we claim to have new enumerative partition results or new bijections proving these results. In fact, paper CS resolves these issues in an even greater generality than we have, and has other applications and

Received by the editors July 24, 2002 and, in revised form, August 30, 2002.

2000 Mathematics Subject Classification. Primary 05A17; Secondary 05A15, 05A19, 11P81.

Key words and phrases. Partition identities, bijections, simple cones. 
further examples. The main point of the paper is to show that the above-mentioned partition results are "natural" from a geometric point of view and that bijections are uniquely defined in this setting.

\section{INTEGER TRIANGLES}

Consider the following classical problem. Let a triple $(a, b, c)$ be an integer-sided triangl 1 if $0 \leq a \leq b \leq c \leq a+b$. Let $\mathcal{A}_{n}$ be the set of integer triangles with perimeter $a+b+c=n$. Then

$$
1+\sum_{n=1}^{\infty}\left|\mathcal{A}_{n}\right| t^{n}=\frac{1}{\left(1-t^{2}\right)\left(1-t^{3}\right)\left(1-t^{4}\right)} .
$$

In other words, the number of integer-sided triangles with perimeter $n$ is equal to the number of partitions of $n$ into parts 2,3 and 4 . The following map $\alpha$ gives an explicit bijection between these two sets: $\alpha\left(2^{x} 3^{y} 4^{z}\right)=(y+z, x+y+z, x+y+2 z)$. Here we use the standard notation $\left(1^{m_{1}} 2^{m_{2}} \ldots\right)$ for partition with $m_{1}$ copies of part (1), $m_{2}$ copies of parts (2), etc. It is crucial in what follows that $\phi$ is linear over $\mathbb{R}$. For more on the the history of the problem and the bijection above, see [A2], A3], and [JWW].

Here is how we approach the problem. Let $\mathbf{A}$ be the set of all triples $(a, b, c) \in \mathbb{R}^{3}$ which satisfy $0 \leq a \leq b \leq c \leq a+b$. Check that $\mathbf{A}$ is a simple cone in $\mathbb{R}^{3}$ with extreme rays given by vectors $v_{1}=(0,1,1), v_{2}=(1,1,1)$, and $v_{3}=(1,1,2)$. Indeed, the inequality $a \geq 0$ follows from $b \leq c \leq b+a$, so $\mathbf{A}$ is the intersection of three halfspaces. Now pairwise intersections of the supporting hyperplanes give us the vectors above.

Observe that the volume $\left|\operatorname{det}\left(v_{1}, v_{2}, v_{3}\right)\right|=1$, i.e. the cone $\mathbf{A}$ is unimodular. Therefore, every integral point $w \in \mathcal{A}:=\mathbf{A} \cap \mathbb{Z}^{3}$ is given by $w=c_{1} v_{1}+c_{2} v_{2}+c_{3} v_{3}$, where $c_{i} \in \mathbb{Z}_{+}:=\{0,1,2, \ldots\}$ (see e.g. [St]). Let $\omega: \mathbb{R}^{3} \rightarrow \mathbb{R}$ be the perimeter function $\omega(a, b, c)=a+b+c$. We conclude that

$$
\sum_{w \in \mathcal{A}} t^{\omega(w)}=\frac{1}{\left(1-t^{\omega\left(v_{1}\right)}\right)\left(1-t^{\omega\left(v_{2}\right)}\right)\left(1-t^{\omega\left(v_{3}\right)}\right)}=\frac{1}{\left(1-t^{2}\right)\left(1-t^{3}\right)\left(1-t^{4}\right)} .
$$

Now consider a simple cone $\mathbf{P}$ of triples $(x, y, z) \in \mathbb{R}^{3}$ which satisfy $x, y, z \geq 0$. The extreme rays are $e_{1}=(1,0,0), e_{2}=(0,1,0)$, and $e_{3}=(0,0,1)$. The integral points $\mathcal{P}:=\mathbf{P} \cap \mathbb{Z}^{3}$ correspond to partitions $\left(2^{x}, 3^{y}, 4^{z}\right)$ into parts 2,3 , and 4 . Obviously, $\left|\operatorname{det}\left(e_{1}, e_{2}, e_{3}\right)\right|=1$ again. Define the partition function $\varphi: \mathbb{R}^{3} \rightarrow \mathbb{R}$ by $\varphi(x, y, z)=2 x+3 y+4 z$. Observe that $\left\{\varphi\left(e_{1}\right), \varphi\left(e_{2}\right), \varphi\left(e_{3}\right)\right\}=\left\{\omega\left(v_{1}\right), \omega\left(v_{2}\right), \omega\left(v_{3}\right)\right\}$ $=\{2,3,4\}$. Therefore, $\sum_{w \in \mathcal{A}} t^{\omega(w)}=\sum_{w \in \mathcal{P}} t^{\varphi(w)}$. Clearly, a linear map defined by $\alpha: e_{i} \rightarrow v_{i}, i=1,2,3$, gives a bijection between $\mathcal{A}_{n}=\{w \in \mathcal{A}: \omega(w)=n\}$ and $\mathcal{P}_{n}:=\{w \in \mathcal{P}: \varphi(w)=n\}=\left\{\lambda=\left(2^{x} 3^{y} 4^{z}\right) \vdash n\right\}$. Furthermore, every linear map $\widetilde{\alpha}: \mathbf{A} \rightarrow \mathbf{P}$ which gives a bijection between $\mathcal{A}_{n}$ and $\mathcal{P}_{n}$ for all $n$ must be equal to $\alpha$ (in fact, restriction to $n=2,3,4$ suffice). Thus it is not surprising that $\alpha$ is the same bijection as defined above. To summarize, we obtain the following result:

Theorem 1. There exists a unique sequence of bijections $\alpha_{n}: \mathcal{A}_{n} \rightarrow \mathcal{P}_{n}$, for all $n \geq 0$, which extends to a linear map $\alpha: \mathbf{A} \rightarrow \mathbf{P}$.

\footnotetext{
${ }^{1}$ For simplicity, we allow here degenerate triangles.
} 


\section{Partitions With NONNEGATIVE $r$-TH DifFERENCES}

Consider a cone $\mathbf{P}_{k} \subset \mathbb{R}^{k}$ of real partitions with at most $k$ parts: $\left(\lambda_{1}, \lambda_{2}, \ldots, \lambda_{k}\right)$, $\lambda_{1} \geq \lambda_{2} \geq \cdots \geq \lambda_{k} \geq 0$. Similarly, consider a cone $\mathbf{P}_{k}^{r} \subset \mathbb{R}^{k}$ of real partitions with at most $k$ parts and nonnegative $r$-th differences:

$$
\begin{aligned}
& \lambda_{1}-\left(\begin{array}{l}
r \\
1
\end{array}\right) \lambda_{2}+\left(\begin{array}{l}
r \\
2
\end{array}\right) \lambda_{3}-\ldots \geq 0, \quad \lambda_{2}-\left(\begin{array}{l}
r \\
1
\end{array}\right) \lambda_{3}+\left(\begin{array}{l}
r \\
2
\end{array}\right) \lambda_{4}-\ldots \geq 0, \ldots, \\
& \lambda_{k-r}-\left(\begin{array}{c}
r \\
1
\end{array}\right) \lambda_{k-r+1}+\left(\begin{array}{l}
r \\
2
\end{array}\right) \lambda_{k-r+2}-\ldots \geq 0 \\
& \lambda_{k-r+1}-\left(\begin{array}{c}
r-1 \\
1
\end{array}\right) \lambda_{k-r+2}+\left(\begin{array}{c}
r-1 \\
2
\end{array}\right) \lambda_{k-r+3}-\ldots \geq 0, \ldots, \\
& \lambda_{k-2}-2 \lambda_{k-1}+\lambda_{k} \geq 0, \quad \lambda_{k-1}-\lambda_{k} \geq 0, \quad \lambda_{k} \geq 0 .
\end{aligned}
$$

These $k$ inequalities define a simple cone $\mathbf{P}_{k}^{r} \subset \mathbb{R}^{k}$ with extreme rays given by vectors $v_{i}=\left(\left(\begin{array}{c}r+i-2 \\ r-1\end{array}\right), \ldots,\left(\begin{array}{c}r+1 \\ r-1\end{array}\right),\left(\begin{array}{c}r \\ r-1\end{array}\right), 1,0\right), 1 \leq i \leq k-1$, and $v_{k}=(1,1, \ldots, 1)$. Indeed, simply check that these vectors lie in the intersection of all but one hyperplane, corresponding to the respective inequality. Clearly, $\left|\operatorname{det}\left(v_{1}, \ldots, v_{k}\right)\right|=1$, so $\mathbf{P}_{k}^{r}$ is unimodular. Thus all integer partitions with $\leq k$ parts and nonnegative $r$-th differences $\lambda \in \mathcal{P}_{k}^{r}:=\mathbf{P}_{k}^{r} \cap \mathbb{Z}^{k}$ are nonnegative linear combinations of $v_{i}$.

Let $\gamma: \mathbb{R}^{k} \rightarrow \mathbb{R}$ be given by $\gamma\left(\lambda_{1}, \ldots, \lambda_{k}\right)=\lambda_{1}+\cdots+\lambda_{k}$. We conclude that

$$
\sum_{\lambda \in \mathcal{P}_{k}^{r}} t^{\gamma(\lambda)}=\prod_{i=1}^{k} \frac{1}{1-t^{\gamma\left(v_{k}\right)}}=\frac{1}{1-t^{k}} \prod_{i=1}^{k-1} \frac{1}{1-t^{\left(\begin{array}{r}
r-1+i \\
r
\end{array}\right.}}
$$

Consider a vector space $\mathbf{Q}_{k}^{r}$ of real partitions into parts $\left(\begin{array}{c}m \\ r\end{array}\right), 1 \leq r \leq r+k-2$, and $k$. More precisely, consider partitions of the form $\left(\left(\begin{array}{c}r \\ r\end{array}\right)^{x_{1}}\left(\begin{array}{c}r+1 \\ r\end{array}\right)^{x_{2}} \ldots\left(\begin{array}{c}r+k-2 \\ r\end{array}\right)^{x_{k-1}}, k^{z}\right)$, where $x_{i}, z \in \mathbb{R}$, and if $k=\left(\begin{array}{c}m \\ r\end{array}\right)$ for some $m \geq r$, we have two types of part $k$. As before, let $\varphi\left(1^{m_{1}} 2^{m_{2}} 3^{m_{3}} \ldots\right)=m_{1}+2 m_{2}+3 m_{3}+\ldots$ We obtain a linear map $\sigma: \mathbf{P}_{k}^{r} \rightarrow \mathbf{Q}_{k}^{r}$ given by $\sigma\left(v_{i}\right)=\left(\begin{array}{c}r+i-1 \\ r\end{array}\right)$, for $i=1, \ldots, k-1, \sigma\left(v_{k}\right)=(k)$.

Let $\mathcal{P}_{n, k}^{r}=\left\{\lambda \in \mathcal{P}_{k}^{r}: \lambda \vdash n\right\}$, and let $\mathcal{Q}_{n, k}^{r}=\left\{\mu \in \mathcal{Q}_{k}^{r}: \lambda \vdash n\right\}$. From above, map $\sigma$ gives a bijection between $\mathcal{P}_{n, k}^{r}$ and $\mathcal{Q}_{n, k}^{r}$, for every $n>0$. Moreover, if $k \neq\left(\begin{array}{c}m \\ r\end{array}\right)$, such a linear map is unique.

Now let $\mathbf{P}$ be a Banach space of all infinite sequences $\left(\lambda_{1}, \lambda_{2}, \ldots\right), \lambda_{i} \in \mathbb{R}$, and with $\ell_{1}$-norm $<\infty$. Let $\mathbf{P}^{r}$ be a subset of these with all $r$-th differences $\geq 0$. Similarly, let $\mathbf{R}^{r}$ be a Banach space of infinite sequences $\mathbf{x}=\left(x_{1}, x_{2}, \ldots\right)$ with $\varphi(\mathbf{x})=\left(\begin{array}{l}r \\ r\end{array}\right) x_{1}+\left(\begin{array}{c}r+1 \\ r\end{array}\right) x_{2}+\cdots<\infty$, and let $\mathbf{Q}^{r}$ be a cone $\left\{x_{i} \geq 0\right.$ for all $\left.i\right\}$.

Observe that $\mathcal{P}_{n, 1}^{r} \subset \mathcal{P}_{n, 2}^{r} \subset \ldots$ stabilizes to $\overline{\mathcal{P}}_{n}^{r}=\bigcup_{k} \mathcal{P}_{n, k}^{r}$, and, similarly, $\mathcal{Q}_{n, 1}^{r} \subset \mathcal{Q}_{n, 2}^{r} \subset \ldots$ stabilizes to $\overline{\mathcal{Q}}_{n}^{r}=\bigcup_{k} \mathcal{Q}_{n, k}^{r}$. As we mentioned in the Introduction, Andrews's Theorem states that $\left|\overline{\mathcal{P}}_{n}^{r}\right|=\left|\overline{\mathcal{Q}}_{n}^{r}\right|$, for all $r, n>0$. Corteel's bijection is a map between these two sets of partitions. Letting $k \rightarrow \infty$ in the reasoning above, we obtain:

Theorem 2. For every $r \geq 1$, there exists a unique sequence of bijections $\sigma_{n}$ : $\overline{\mathcal{P}}_{n}^{r} \rightarrow \overline{\mathcal{Q}}_{n}^{r}$, for $n \geq 1$, which extend to a linear map $\sigma: \mathbf{P}^{r} \rightarrow \mathbf{R}^{r}$. 


\section{Corteel-Savage type Generalizations AND EXAmples}

In a recent paper [CS the authors obtained an advanced generalization of Andrews's Theorem and Corteel's bijections. Basically, they considered the inequalities of general form $\lambda_{i} \geq a_{i, i+1} \lambda_{i+1}+a_{i, i+2} \lambda_{i+2}+\ldots$ with integer values of $a_{i}$. They also present a number of interesting examples. While our approach extends verbatim to general compositions investigated in CS, we concentrate here on a nice special case of partitions.

Let $\Lambda$ be a set of integer sequences $\lambda=\left(\lambda_{1}, \lambda_{2}, \ldots\right)$, with a finite number of nonzero entries. Fix a nonnegative integer sequence $\left(a_{1}, a_{2}, \ldots, a_{r}\right)$. Now let $\mathcal{A}_{n}$ be a set of partitions $\lambda \vdash n$, such that $\lambda_{i}-\lambda_{i+1} \geq a_{1} \lambda_{i+1}+a_{2} \lambda_{i+2}+\cdots+a_{r} \lambda_{i+r}$. As before, let $\mathcal{A}=\bigcup_{n} \mathcal{A}_{n}$.

For a sequence $\left(b_{1}, b_{2}, \ldots\right)$ with $b_{1}<b_{2}<\ldots$, let $\mathcal{B}$ be a set of partitions into parts $b_{i}$. Let $\mathcal{B}_{n}=\{\mu \in \mathcal{B}: \mu \vdash n\}$. Finally, define Banach spaces $\mathbf{A} \supset \mathcal{A}$ and B $\supset \mathcal{B}$ as in the previous section.

Theorem 3. For every sequence $\left(a_{1}, a_{2}, \ldots\right)$ and a set of partitions $\mathcal{A}$, defined as above, there exists a sequence $\left(b_{1}, b_{2}, \ldots\right)$ and a set of partitions $\mathcal{B}$ into parts $b_{i}$, such that $\left|\mathcal{A}_{n}\right|=\left|\mathcal{B}_{n}\right|$ for all $n \geq 1$. Furthermore, there exists a unique sequence of bijections $\sigma_{n}: \mathcal{A}_{n} \rightarrow \mathcal{B}_{n}$, which extend to a linear map $\sigma: \mathbf{A} \rightarrow \mathbf{B}$.

The proof follows the same framework as before, so we shall just point out the differences. Start by defining $c_{1}=1, c_{0}=c_{-1}=c_{-2}=\cdots=0, c_{i+1}=$ $\left(1+a_{1}\right) c_{i}+a_{2} c_{i-1}+a_{3} c_{i-2}+\ldots$ Computing extremal rays gives us, sequentially, $v_{1}=\left(c_{1}, 0,0,0, \ldots\right), v_{2}=\left(c_{2}, c_{1}, 0,0, \ldots\right), v_{3}=\left(c_{3}, c_{2}, c_{1}, 0, \ldots\right)$, etc. Since $a_{i}$ are nonnegative integers, vectors $v_{i}$ are integer partitions. Setting $b_{i}=c_{1}+\ldots+c_{i}$ gives us the desired set of partitions $\mathcal{B}$. The rest of the proof follows verbatim.

Example 0. Let $\mathcal{A}_{n}$ be the set of all partitions $\lambda \vdash n$ which corresponds to the trivial sequence $(0)$. Then $v_{i}=(1,1, \ldots, 1,0, \ldots, 0)$ with $i$ ones, and $c_{i}=i$. In this case $\mathcal{B}_{n}=\mathcal{A}_{n}$, and the map $\sigma: \mathcal{A}_{n} \rightarrow \mathcal{B}_{n}$, defined by $\sigma_{n}: v_{i} \rightarrow(i)$, is a simple conjugation $\sigma(\lambda)=\lambda^{\prime}$ (see [A1]). Thus our geometric approach shows that conjugation is a unique automorphism on the set of partitions which translates part lengths into part multiplicities.

Example 1. Let $\mathcal{A}_{n}$ be the set of partitions $\lambda \vdash n$, such that $\lambda_{i} \geq r \lambda_{i+1}$ for all $i$. This corresponds to a sequence (1). In this case $\mathcal{B}_{n}$ is the set of partitions $\mu \vdash n$ into parts $b_{i}=\left(r^{i}-1\right) /(r-1)$. The corresponding bijection $\tau_{n}: \mathcal{B}_{n} \rightarrow \mathcal{A}_{n}$ is given by $\tau:\left(b_{i}\right) \rightarrow\left(r^{i-1}, r^{i-2}, \ldots, r, 1\right)$. This bijection is due to Hickerson (see also CS], Example 4). Our approach shows that it is unique in the same sense as before.

Example 2. Let $\mathcal{A}_{n}$ be the set of partitions $\lambda \vdash n$, such that $\lambda_{i} \geq \lambda_{i+1}+\lambda_{i+2}$, for all $i$. This corresponds to a sequence $(0,1,1)$. From Theorem 3 , we obtain the set $\mathcal{B}_{n}$ of partitions into parts $\left\{1,2,4,7,12, \ldots, F_{k}-1, \ldots\right\}$, where $F_{k}$ is the $k$-th Fibonacci number. The corresponding bijection $\tau_{n}: \mathcal{B}_{n} \rightarrow \mathcal{A}_{n}$ is given by $\tau:\left(F_{k}-1\right) \rightarrow\left(F_{k-1}, F_{k-2}, \ldots, 2,1\right)$ and is given in [CS], Example 6 .

To generalize the examples, let $A(t)=a_{1} t+a_{2} t^{2}+\ldots, B(t)=1+b_{1} t+b_{2} t^{2}+\ldots$, and $C(t)=1+c_{1} t+c_{2} t^{2}+\ldots$. Now the recurrence relations give us $C(t)=$ $1+t C(t)+A(t) \cdot C(t)$ and $B(t)=C(t) /(1-t)$. We conclude that

$$
B(t)=\frac{1}{(1-t)(1-t-A(t))} \text {. }
$$


This suggests a number of other examples, some of which are not formally implied by either Theorem 3 or [CS], but follow with no change in the proof.

Example 3. Let $\mathcal{A}_{n}$ be the set of partitions $\lambda \vdash n$, such that successive 1-st differences weakly decrease: $\lambda_{i}-\lambda_{i+1} \geq \lambda_{i+2}-\lambda_{i+3}$, for all $i$. This corresponds to a sequence $(0,1,-1)$. From above, $B(t)=1 /(1-t)^{2}\left(1-t^{2}\right)$. Therefore, $\left|\mathcal{A}_{n}\right|$ is equal to $\left|\mathcal{B}_{n}\right|$ the number of partitions of $n$ into quarter-squares $\left\lfloor\frac{m^{2}}{4}\right\rfloor$, i.e. into parts $\{1,2,4,6,9,12,16,20, \ldots\}$ (see [Sl], Sequence A002620). Depending on the parity, the corresponding bijection $\tau_{n}: \mathcal{B}_{n} \rightarrow \mathcal{A}_{n}$ maps these parts into partitions $(k, k-1, k-1, \ldots, 2,2,1,1)$ and $(k, k, k-1, k-1, \ldots, 2,2,1,1)$. We refer to Sl] for references to several combinatorial interpretations of quarter-squares.

Example 4. Let $\mathcal{A}$ be the set of partitions $\lambda$, such that for all $i \geq 1$,

$$
\lambda_{i} \geq \lambda_{i+1}+2 \lambda_{i+2}+5 \lambda_{i+3}+\ldots+c_{k} \lambda_{i+k}+\ldots,
$$

where $c_{k}=\frac{1}{k+1}\left(\begin{array}{c}2 k \\ k\end{array}\right)$ are Catalan numbers. Since $1+A(t)=C(t)$ in this case, we have $b_{n}=1+2+5+\ldots+c_{n}$. Therefore, the corresponding bijection $\tau_{n}: \mathcal{B}_{n} \rightarrow \mathcal{A}_{n}$ maps $\left(b_{k}\right)$ into $\left(c_{n}, c_{n-1}, \ldots, 5,2,1\right)$. Naturally, Catalan numbers can be uniquely defined this way.

Example 5. Let $\mathcal{A}$ be the set of partitions $\lambda$, such that for all $i \geq 1$,

$$
\lambda_{i} \geq \lambda_{i+1}+\lambda_{i+2}-\lambda_{i+5}-\lambda_{i+7}+\lambda_{i+12}+\lambda_{i+15}-\lambda_{i+22}-\lambda_{i+26}+\ldots,
$$

where the general terms are $(-1)^{k-1} \lambda_{i-m}$, and $m=\frac{k(3 k \pm 1)}{2}$ are pentagonal numbers. Let $\mathcal{B}$ be the set of partitions into parts $b_{k}=1+p(1)+p(2)+\cdots+p(k-1)$, where $p(m)$ is the number integer of partitions of $m$. Then $\left|\mathcal{A}_{n}\right|=\left|\mathcal{B}_{n}\right|$, for all $n$. This follows from Euler's Pentagonal Theorem (see e.g. A1]) and the same reasoning as above. The corresponding vectors are $v_{k}=(p(k-1), p(k-2), \ldots, 7,5,3,2,1,1)$. We leave the details to the reader.

\section{Final Remarks}

Note that Theorem 3 does not generalize Theorem 2, as the corresponding sequence contains negative binomial coefficients. Examples 3 and 5 do not follow from Theorem 3 as well. We leave the most general version of the theorem to the reader (see [CS] for the numerical version).

While Catalan numbers are fixed points of $1+A(t) \rightarrow C(t)$ transformation, a sequence A007317 in [S] is a fixed point of $1+A(t) \rightarrow B(t)$ transformation. We omit the corresponding bijection and combinatorial interpretations.

It is rather rare to see a bijection uniquely determined in a combinatorial problem. In fact, we cannot recall a single such example in the context of Partition Theory. Heuristically, the uniqueness of a bijection comes from taking all sets $\mathcal{A}_{n}$ together and treating them as a single object $\mathcal{A}$, with linearity conditions relating $\mathcal{A}_{n}$ for different $n$. It would be nice to find less trivial examples of this phenomena.

The connection between partitions and integer points was noted earlier in several special cases (see e.g. $\mathrm{BE}$ ), but have never been rigorously studied. We hope that this paper will open a way to further connections between Partition Theory and Discrete Geometry. 


\section{ACKNOWLEDGEMENTS}

We would like to thank the referee for pointing out paper [CS]. Neil Sloane showed us sequences A007317 and A014137 in Sl. We are grateful to Richard Stanley for encouragement. The author was partially supported by the NSA and the NSF.

\section{REFERENCES}

[A1] G. E. Andrews, The Theory of Partitions, Addison-Wesley, Reading, MA, 1976. MR 58:27738

[A2] G. E. Andrews, A note on partitions and triangles with integer sides, Amer. Math. Monthly 86 (1979), 477-478. MR 80g:10009

[A3] G. E. Andrews, MacMahon's Partition analysis. II. Fundamental theorems, Ann. Combin. 4 (2000), 327-338. MR 2002g:05014

[APR] G. E. Andrews, P. Paule, A. Riese, MacMahon's partition analysis. III. The Omega package, European J. Combin. 22 (2001), 887-904. MR 2002h:11100

[BE] M. Bousquet-Mélou, K Eriksson, Lecture Hall Partitions 2, Ramanujan J. 1 (1997), 165185. MR 99c:05016

$[\mathrm{CCH}]$ R. Canfield, S. Corteel, P. Hitczenko, Random partitions with non-negative $r$-th differences, Adv. in Appl. Math. 27 (2001), 298-317. MR 2002j:05012

[CS] S. Corteel, C. Savage, Partitions and Compositions defined by inequalities, Ramanujan J. (to appear).

[H] D. R. Hickerson, A partition identity of the Euler type, Amer. Math. Monthly 81 (1974), 627-629. MR 49:2526

[JWW] J. H. Jordan, Ray Walch, R. J. Wisner, Triangles with integer sides, Amer. Math. Monthly 86 (1979), 686-689. MR 81f:05013

[Sl] N. J. A. Sloane, On-line Encyclopedia of Integer Sequences, available at $h t t p: / / w w w$. research.att.com/njas/sequences. Electron. J. Combin. 1 (1994). MR 95b:05001

[St] R. P. Stanley, Enumerative combinatorics, Vol. 1, Cambridge University Press, Cambridge, 1997. MR 98a:05001

[Z] D. Zeilberger, Sylvie Corteel's One-Line Proof of a Partition Theorem Generated by Andrews-Paule-Riese's Computer, Shalosh B. Ekhad's and Doron Zeilberger's Very Own Journal, available at: http://www.math.rutgers.edu/ zeilberg/mamarim/mamarimhtml/ corteel.html.

Department of Mathematics, Massachusetts Institute of Technology, Cambridge, MASSACHUSETTS 02139

E-mail address: pak@math.mit.edu 